\title{
CONTROL DE ARMAS EN EL NUEVO FEDERALISMO AMERICANO
}

\author{
JOSÉ MANUEL MARTÍNEZ SIERRA \\ Profesor Titular de Derecho Constitucional UCM \\ Professor of European Union Law and Government Chair \\ Universidad de Harvard \\ JUAN MANUEL MECINAS MONTIEL \\ Catedrático Conacyt \\ Centro de Investigación y Docencia Económicas
}

SUMARIO

I. Introducción. II. Las bases y el desarrollo de la Segunda Enmienda. III. United States v. Lopez. IV. Heller: Volviendo a la Segunda Enmienda. V. Conclusiones.

\section{INTRODUCCIÓN}

Los últimas matanzas en los Estados Unidos de América y su consiguiente impacto en la pugna presidencial norteamericana han vuelto a poner de actualidad un tema muy sensible para el pueblo americano como es el «control de armas». Esta cuestión es relevante no solamente por el impacto que tiene la perdida de toda vida humana, sino porque estas muertes están directamente vinculadas a un problema de naturaleza constitucional que, por otra parte es prácticamente inexistente, tanto en el fondo como en la forma, en la mayoría de los Estados del continente Europeo.

El tema que nos ocupa en estas páginas tiene, como se sabe, una trascendencia constitucional innegable. En primer lugar, en él entran en juego los derechos y libertades tanto de los sujetos activos como de los sujetos pasivos de quienes se ven envueltos en los tiroteos descontrolados o perpetrados por francotiradores. En segundo lugar, junto a esta afectación directa, aparece también la cuestión de la seguridad pública. A este respecto, y con independencia de la afectación constitucional transversal manifestada por múltiples vías, el nuevo constitucionalismo proyectará sobre dicho asunto un peso creciente, dado que desde el 2001 la segu- 
ridad pública y su garantía son ejes medulares de las políticas públicas en la sociedad mundial. Y, en tercer lugar, siendo esta dimensión particularmente importante en el debate que nos ocupa dentro del constitucionalismo americano, afecta ciertamente a la distribución vertical de competencias entre la Federación y los Estados. Todos estos aspectos, por venir ligados a una problemática de ámbito global y de naturaleza transfronteriza — entre estados de la Unión y allende las fronteras americanas-, demanda una reflexión tanto sobre las virtudes de federalizar competencias para resolver ciertas cuestiones, como sobre el impacto en la ortodoxia y la tradición constitucional americana.

El control de armas en los Estados Unidos encuentra su génesis en el propio nacimiento del país. En este sentido, es indudable que la interpretación originalista de la Constitución americana, con todo su carácter conservador, se manifieste triunfante, al menos de forma coyuntural, en un bien entrado siglo XXI, porque en la discusión no se miran los fines en relación con la situación actual, sino los fines en relación con las restricciones y límites que se previeron durante la aprobación de la Segunda Enmienda de la carta magna americana a finales del siglo XVIII. Se trata de una visión en la que el derecho de los individuos a portar armas y su inmutabilidad proyectada hacia el presente llama la atención, más si cabe, si se considera que desde la aprobación de la Segunda Enmienda hasta nuestros días se pueden identificar largos periodos de inestabilidad militar, constitucional y política, además de una espiral creciente de violencia con armas de fuego.

Es preciso apuntar como premisa que el debate en relación al control de armas en los Estados Unidos es un debate sesgado, simplemente porque está lleno de falacias muy consolidadas en un amplio sector de la población americana ${ }^{1}$. Las distintas posturas sobre la cuestión aquí abordada resultan irreconciliables; de hecho, su defensa parece ser un ejercicio de suma cero, si se tiene en cuenta que los defensores de la posesión de armas temen que cualquier intento de control sea el detonante que conduzca más tarde o más temprano a una prohibición total.

Para ordenar este debate desde una perspectiva constitucional, en el presente trabajo se analizan dos sentencias (Heller y López) dictadas por el Tribunal Supremo de los Estados Unidos de América (TS) para tratar de entender, de un lado, el impacto constitucional de la cuestión y, de otro, el impacto del nuevo federalismo, tal y como lo está entendiendo el TS, a saber: como preeminencia de la soberanía de los Estados. Se trata de analizar qué impacto tiene el entendimiento del federalismo de la forma en que lo hace el TS, cuando se enfrenta con una mayor o menor restricción en la tenencia y porte de armas. Con carácter previo al análisis de los dos casos mencionados, dedicaremos un apartado a recordar la génesis y evolución legislativa y constitucional de la cuestión. Finalmente, tras los tres apartados de análisis concluiremos a modo de recapitulación intentando lle-

1 Rhetoric of Gun Control, The [article] American University Law Review, Vol. 42, Issue 1 (1992), pp. 53-114; Mcclurg, A., J. 42 Am. U. L. Rev. 53 (1992). 
var a cabo una prognosis de la situación que se vislumbra tras la pugna por la Presidencia entre Clinton y Trump que se avecina.

\section{LAS BASES Y EL DESARROLLO DE LA SEGUNDA ENMIENDA}

La Segunda Enmienda es el punto medular sobre el que pivota la discusión constitucional y política sobre el control de armas en Estados Unidos, pues se discute precisamente si dicha enmienda reconoce o no un derecho individual a la posesión y porte de armas.

Como se sabe «el derecho a la poseer y portar armas» es parte de las diez primeras enmiendas a la Constitución Americana, que se conocen comúnmente como el Bill of Rights (americano). Y es precisamente del Bill of Rights ${ }^{2}$ de la antigua metrópolis británica de 1689 de donde toma su origen la Segunda Enmienda. Conviene, no obstante, precisar que en ningún momento se puede inferir del texto británico, que en virtud del mismo se permita portar armas o que su posesión se pueda concebir como un derecho. De hecho, la discusión actual sobre el alcance de la Segunda Enmienda no es una cuestión contemporánea, es por contra otro episodio en una constante. Así, por ejemplo, en 1990 Sanford Levinson —uno de los constitucionalistas americanos más respetados - la tildaba como una «enmienda vergonzosa», un «remiendo de texto en las conservaciones constitucionales ${ }^{3}$.

En la etapa fundacional de los Estados Unidos era común la tenencia de armas de fuego, y los estados de la Unión regulaban su tenencia en aras de la seguridad pública ${ }^{4}$. Ello fue un elemento medular en su constitucionalización y en su desarrollo inicial. En 1866, el TS decidió que la Segunda Enmienda fuera oponible a los Estados, lo que «abre la posibilidad de que tenga un significado distinto si es oponible a las leyes estatales que cuando es oponible a las leyes federales» 5 . Con dicho fallo judicial se produjo un salto cualitativo, pues no se trataba únicamente de la posesión de armas sino de la posibilidad de portarlas, y ya no vinculado a formar parte de una milicia que defendiera a la nación frente a la opresión, sino, en términos de los Estados Unidos en la época de la pos-guerra civil, para asegurar el derecho a la autodefensa de los hombre libres. Sirva aquí la pluma de Meltzer: «This distinction made no difference in Heller and McDonald because the Court found that the 1791 and the 1866 understandings of the Second Amendment both guaranteed an individual right. ${ }^{136}$ But that uniformity might

2 El documento británico de 1689 establece «subjects who are Protestants may bear arms for their defence as permitted by law».

3 Levinson, S., «The embarrasing Second Amendment», en Yale Law Journal, Volumen 99, pp. 637-65.

4 Meltzer, J., Open Carry for All: Heller and Our Nineteenth-Century Second Amendment, en Yale Law Journal, Vol. 123, n. ${ }^{\circ}$, pp. 1118-1625.

5 Ibídem. 
not remain in a case involving the right to carry. Indeed, the Court described the post-Civil War Second Amendment right in $\mathrm{McD}$ onald as one that was no longer associated with the militia but instead intended to ensure the right to self-defense of the freedmen. It is not difficult to imagine a more robust right to carry as part of this later understanding» ${ }^{6}$.

Tras los momentos históricos indicados y hasta los recientes pronunciamientos judiciales que estudiaremos posteriormente, la Segunda Enmienda ha sido modulada en pocas ocasiones por el TS de los Estados Unidos. La primera gran decisión en el siglo Xx que se tomó en relación a ella fue en United States v. Miller ${ }^{7}$. En dicho caso, el criminal Jack Miller fue arrestado en 1938 y acusado de violar la NFA (National Firearm Act), aunque el Tribunal de Distrito desestimó los cargos en su contra y tuvo que ser el Tribunal Supremo quien determinara en 1939 que la Segunda Enmienda no garantiza ningún derecho a portar y poseer escopetas recortadas.

Las cifras relativas a las armas que se encuentran en hogares americanos, las muertes de civiles por arma de fuego y los tiroteos masivos no han dejado de crecer escandalosamente. Desde $2004^{8}$, han habido 47 tiroteos y han muerto 411 personas; solo en la primera mitad de 2016 hubo 61 muertos en tiroteos masi$\operatorname{vos}^{9}$, sin que exista un solo ejemplo de prohibición de porte de arma de fuego en los parlamentos estatales ${ }^{10}$. Se estima que en Estados Unidos hay más puntos de venta de armas que oficinas de correos. Para poder comprar un arma, el comprador se somete a una revisión de sus antecedentes criminales que normalmente tarda escasos minutos. En los Estados Unidos, un país con poco más de 300 millones de habitantes, existen aproximadamente 300 millones de armas.

Junto con la dramática situación que se vive dentro de las fronteras de los Estados Unidos, el debate nacional, incluso los más autorizados, ignora normalmente el gran negocio que significa la venta de armas a México y otros países con conflictos armados y delincuencia organizada masiva, lo cual implica un problema mayúsculo global al haber un descontrol, a veces deseado y en ocasiones inesperado, respecto de las armas en circulación.

El descontrol es tal que la venta de armas fuera del país termina afectando también a ciudadanos estadounidenses, desmontando así la interesada ceguera de los que afirman que los problemas globales, con afectación en los Estados Unidos,

6 Ídem.

7 United States v. Miller, 307 U.S. 174 (1939).

8 Es el año en el que quedó sin vigencia la Violent Crime Control and Law Enforcement Act de 1994, que prohibía la posesión, transferencia o manufactura de rifles de asalto semiautomático.

9 A ello hay que añadir la cifra de lesionados. Por ejemplo, el 12 de junio del 2016, en un tiroteo en Orlando, Florida, Omar Mateen mató a 49 personas y dejó un total de 53 heridos, en ataque que ocurrió en el centro nocturno Pulse. Véase The Washington Post, «Minute by minute: How the attack in Orlando unfolded», en The Washington Post, 1 de agosto de 2016, disponible en https://www.washingtonpost.com/graphics/ national/orlando-shooting/, revisado el 15 de agosto de 2016.

10 Véase http://apps.bostonglobe.com/graphics/2016/06/make-it-stop/, revisado el 29 de agosto de 2016. 
se resuelven con medidas adoptadas exclusivamente dentro de las fronteras ${ }^{11}$. El caso paradigmático de lo afirmado se encuentra en el programa «Rápido y Furioso» ${ }^{12}$, que tenía como objetivo rastrear la ruta que seguían las armas desde los vendedores callejeros hasta los jefes de carteles mexicanos. Muchas de esas armas se encontraban en Estados Unidos ${ }^{13}$ y otras fueron introducidas a territorio mexicano, pero terminaron siendo usadas contra ciudadanos norteamericanos, como los agentes Brian Terry (asesinado el 14 de diciembre de 2010) —quien fue herido mortalmente con una $\mathrm{AK}-47$ semiautomática, cuyos números de serie concordaban con armas compradas para el programa «Rápido y Furioso» ${ }^{14}$ - y Orlando Zapata, asesinado en febrero de 2011 con una AK-47 semiautomática comprada en Texas ${ }^{15}$.

Ante dicha realidad, en el ámbito público, las posiciones van desde los que afirman que en los Estados en los que se permite portar armas existen menos muertes y menos heridos por ataques multi-homicidas con armas de fuego ${ }^{16}$, hasta los que pugnan por una prohibición de tenencia y porte de armas con mayor o menor rigidez ${ }^{17}$.

En la tensión del debate sobre la cuestión que nos ocupa, como hemos adelantado, incluso la mayoría que está a favor del mayor control lo hace de forma muy limitada. Por ello, procede destacar la distinción entre la posesión de armas abiertamente en público (mostrándolas) y una posesión en público encubierta (donde las armas estén ocultas). Trece Estados de la Unión exigen que se tenga un permiso especial para portar armas abiertamente, mientras que seis Estados prohíben dicha práctica, lo que nos deja a un total de treinta y un Estados en los que no existe impedimento alguno para portarlas de manera visible ${ }^{18}$. Sin embargo, en los cincuenta Estados de la Unión Americana existen leyes que permiten

11 Camisón, J.A., «Global Ferderalism: A Solution for the Global Economic Crisis», en LóPEZ-BASAGURen, A. (eds.), The ways of federalism in Western Countries and the Horizonts of Territorial Autonomy in Spain, Vol. 1, Heidelberg, Springer-Verlag, 2013.

12 Horwitz, S., «Operation Fast and Furious: A gunrunning sting gone wrong», The Washington Post, 26 de julio de 2011. Horwitz afirmó que se habían comprado màs de dos mil armas para el programa «rápido y furioso», y que sólo que habían recuperado cerca de seiscientas.

13 Ibídem.

14 Ibídem.

15 Воотн, W., «Jaime Zapata's death: One year later, still many unanswered questions», The Wasbington Post, 16 de febrero de 2012, disponible en https://www.washingtonpost.com/blogs/blogpost/post/ jaime-zapatas-death-one-year-later-still-many-unanswered-questions/2012/02/16/gIQAPtH0HR_blog. html, revisado el 27 de agosto de 2016.

16 Lott Jr., J. y Landes, W., Multiple Victims Public Shootings, 1996, disponible en http://dx.doi. org/10.2139/ssrn.272929, revisado el 20 de agosto de 2016.

17 Kopel, D., «The Great Gun Control War of the 20th Century - And its Lessons for Gun Laws Today» (February 2, 2013). Fordham Urban Law Journal, vol. 39, n. ${ }^{\circ}$ 5, pp. 1527-1666 (October 2012). Disponible en: http://ssrn.com/abstract $=2070925$

18 Molla, R. Map: «Where is an “open carry” legal?», The Wall Street Journal, 22 de agosto de 2014, disponible en http://blogs.wsj.com/numbers/map-where-is-open-carry-legal-1715/, revisado el 25 de agosto de 2016. 
portar armas en público siempre que estén escondidas. En el mismo sentido, son pocos los Estados donde existen criterios (entre ellos destaca el criterio de «buena razón») para portar un arma.

En este escenario de galimatías legislativo, la opinión y decisión de los juzgadores es vital. La discusión legal más reciente y de mayor relevancia se presentó en el Tribunal de Apelaciones del Noveno Circuito que decidió el caso Peruta vs San Diego County, en el que determinó que no existe un derecho protegido por la segunda enmienda para portar armas de fuego escondidas en público. Peruta y otros alegaron, por su parte, que les fue negado el permiso para portar un arma escondida porque no probaron el requisito de «buena razón» para portar armas, y el Tribunal de Apelaciones determinó que al no haber un derecho protegido por la Segunda Enmienda para portar armas que no estuvieran a la vista, ello era suficiente para declarar cualquier prohibición o requerimiento como constitucional ${ }^{19}$.

Esto deja la puerta abierta a una nueva interpretación por parte del Supremo Americano respecto a la posesión de armas en público. El Tribunal ha sufrido cambios importantes en su integración durante los últimos lustros, hecho este que ha impactado en sus interpretaciones constitucionales. Con la llegada de Reagan a la presidencia de los Estados Unidos, el asalto conservador al Supremo Americano se hizo más patente, por más que el histórico rechazo a la candidatura de Robert Bork fuera un momento en que no quedaba claro el rumbo de ese intento conservador. La nueva orientación conservadora tenía tres objetivos fundamentales. En primer lugar, que el derecho a portar armas se mantuviera incólume, lo implicaba también una jurisprudencia acorde sobre control de armas. En segundo, lograr un giro conservador en los casos sobre aborto a través de una suerte de overruling respecto de Roe $v$. Wade. Y, en tercer lugar, un nuevo pronunciamiento sobre la affirmative clause, respecto del caso Brown. Con la llegada de Alito y Roberts al TS a propuesta del republicano George W. Bush, las condiciones fueron inmejorables y el año 2008 marcó el primer punto importante del innegable giro conservador del Tribunal Supremo y sus sentencias. En este sentido, el caso Heller se presentaba como la oportunidad de oro para zanjar la discusión sobre el alcance del derecho de los ciudadanos a portar y tener armas de fuego.

No obstante lo anterior, el actual escenario político y social ha cambiado, las masacres con armas de fuego son cada vez más una constante, además de que con la muerte del magistrado conservador Antonin Scalia, por primera vez en sesenta años el Supremo podría tener una mayoría liberal, justo en el momento en el que un Presidente demócrata ocupa la casa Blanca, y después de que las últimas dos magistradas nombradas — Sotomayor y Keagan — se han integrado en el ala li-

19 United States Court of Appeals of the Ninth Circuit, Peruta et al v. County of san Diego n. ${ }^{\circ} 10$ 56971, p. 46. 
beral del máximo Tribunal americano. La nominación de Merrick Garland para sustituir a Scalia, y una probable victoria de Hillary Clinton en las elecciones presidenciales de noviembre de 2016, podrían cambiar muy sensiblemente el mapa de mayorías en el seno del Supremo estadounidense.

Merrick Garland ${ }^{20}$ fue propuesto por Barack Obama para ser el nuevo magistrado del Tribunal Supremo de los Estados Unidos de América el 16 de marzo de 2016. Seis meses después, Garland no ha sido sometido al procedimiento de audiencias que lleva a cabo el Senado en el marco del proceso de nombramiento, toda vez que los Republicanos consideran que la propuesta de un nuevo juez para ocupar la vacante ante la muerte de Antonin Scalia, debe recaer en el próximo presidente de los Estados Unidos que saldrá, como se sabe, de la contienda entre Donald Trump y Hillary Clinton y, obviamente, los Republicanos esperan que Trump sea el ganador y con ello evitar que se abra una nueva época liberal (la primera en los últimos 60 años) en el Tribunal Supremo Americano.

En realidad, los argumentos para rechazar de manera informal la propuesta de Obama son fundamentalmente políticos, y la preocupación principal es que Garland se una al bloque liberal, lo que podría conducir a abrir la puerta a una nueva lectura, entre otras, de la Segunda Enmienda, en el sentido de que se restrinja la posibilidad de tenencia y posesión de armas de fuego en público. El mismo Donald Trump, en un lamentable discurso pronunciado el 9 de agosto de 2016, sostuvo públicamente que solo los defensores de la Segunda Enmienda pueden parar a Hillary Clinton en su intención de nombrar a jueces del Tribunal Supremo que impongan una visión más restrictiva del derecho a portar armas. Es oportuno señalar que Trump fue deliberadamente confuso en su intervención, como denunciaron destacados republicanos, pues no estaba del todo claro si para parar a Clinton se refería exclusivamente al uso del poder del voto ${ }^{21}$.

Cabe aclarar, además, que no se trata exclusivamente de una lucha entre Republicanos y Demócratas, pues tanto unos como otros albergan en su seno seguidores de una restricción, de mayor o menor calado, de la Segunda Enmienda; también se trata de un debate relativamente nuevo, el cual gira alrededor de la Gun Control Act de 1968, y que encuentra en Heller y en López nuevas plataformas de discusión como pasamos a analizar de forma inmediata.

Los límites de la discusión en relación a la tenencia y porte de armas se han abordado tradicionalmente como problemas concernientes a la veracidad o falsedad de que la Segunda Enmienda prohíba o garantice la tenencia de un arma. Solo a partir de Columbine - el primer asesinato masivo con arma de fuego en una escuela- se comenzó a discutir, aunque de manera ciertamente tenue, como un

20 Véase, «Merrick Garland, President obama's sensible Supreme Court choice» en The New Yorker, 16 de Marzo de 2016, disponible en http:/www.newyorker.com/news/news-desk/president-obamas-sensible-supreme-court-choice, revisado el 14 de julio de 2016.

21 Corasaniti, N. Haberman, M., «Trump Suggests Gun Owners Act Against Clinton», en The New York Times, 10 de agosto de 2016, p. A1. 
problema de violencia a gran escala vinculada al armamento. La cuestión es distinta: en la discusión entre tirios y troyanos sobre la interpretación que se le debe dar a Heller — más allá del claro giro conservador del TS y de la llegada triunfal de Scalia a la cima del control de armas con sus tesis originalistas - no está claro si los críticos de la visión pro armas en realidad ganarán algo más que el debate si el TS decide un nuevo caso — tal vez con Garland como magistrado- en el que considere una visión más restrictiva de la Segunda Enmienda. El gran cambio en estos momentos consistiría en centrar la discusión en términos de violencia generada a partir de la tenencia de armas. Una visión de este estilo haría no depender el debate de una sola condición: la mayoría liberal o conservadora en el Supremo Americano, lo que hasta ahora prevalece, como se verá a continuación.

\section{UNITED STATES V. LOPEZ}

Sin duda alguna, el caso más sonado en los últimos años en el constitucionalismo americano, por lo que a cuestiones de federalismo se refiere, es el conocido como caso Lopez ${ }^{22}$, aunque podemos decir que es corolario o eslabón (está por verse) de todo un movimiento conservador que ha inundado el Tribunal Supremo de los Estados Unidos en los últimos tiempos.

Lopez ha sido considerado por algunos como un hito ${ }^{23}$, aunque otros minimizan su importancia, sobre todo por los efectos no tan claros que parece tener en la concepción del federalismo y de la distribución de competencias entre los Estados y la federación ${ }^{24}$. Lopez es un caso polifacético que engloba muchos y muy distintos problemas: la interpretación de la commerce clause ${ }^{25}$, federalismo ${ }^{26}$, separación de poderes $^{27}$, el rol del Tribunal Supremo en el sistema político ${ }^{28}$. Y todo ello ha dado lugar a múltiples estudios ${ }^{29}$.

22 United States v. Lopez US 514 (549) 1994.

23 Según la opinión de B. Ackerman. Cit. en Fedarko, K., «A gun ban is shot down» en Time, 24 de enero de 2001. Disponible en http:/www.time.com/time/magazine/article/0,9171,134119,00.html

$24 \mathrm{Al}$ respecto «The Lesson of Lopez: The political dynamics of federalism's political safeguards», en Harvard Law Review, vol. 119, pp. 609-630.

25 Al respecto, véase Harvard Law Review, vol. 119, n. ${ }^{\circ}$ 169, 2005, pp. 169-178.

26 Véase MANNING, J., «Federalism and the generality problem in constitutional interpretation», en Harvard Law Review, vol. 122, n. ${ }^{\circ} 8$, junio 2009, pp. 2009-2069.

27 Una visión moderna, con un toque de resumen se puede encontrar en ACKerman, B. La nueva división de poderes, México: FCE, 2007.

28 El recientísimo libro del magistrado Breyer da luz sobre el papel que ha jugado y el papel que debería jugar. Breyer, Stepehn. Making our democracy work. A judge's view, NY: Knopf, 2010., También FArBer et al., Cases and Material on constitutional law: themes for the constitution third century, St. Paul, MN: West, 2009, cap. I.

29 «The Lesson of Lopez: The political dynamics of federalism's political safeguards», en Harvard Law Review, vol. 119, pp. 609-630; MANNING, J., «Federalism and the generality problem in constitutional interpretation", en Harvard Law Review, vol. 122, n. ${ }^{\circ} 8$, junio 2009, pp. 2009-2069; PALMER y LAVERTY. "The impact of Lopez on intergovernmental relations: a preliminary assesstment», en Publius, vol. 26, 
El caso fue planteado como un problema de inconstitucionalidad de un statute law que prohibía portar armas en zonas escolares, lo que Alfonso Lopez sostenía que era inconstitucional porque la ley (statute), con base en la cual se le acusaba, había sido emitida por una autoridad que carecía de competencia para ello (el Congreso de los Estados Unidos de América), pues la regulación contenida en el statute quedaba fuera de la commerce clause, en base a la cual se permite al Congreso federal norteamericano legislar sobre un sinfín de materias, de acuerdo al artículo 1 sección 8 de la Constitución Americana, tal y como el mismo TS ha definido desde el caso Gibbons ${ }^{30}$.

Lopez tuvo eco por lo delicado del tema (portar armas en una escuela) y por sus implicaciones para el federalismo. Los hechos de los que trae causa la sentencia son los siguientes. Alfonso López era un estudiante que portaba un arma en una escuela en Texas (supuestamente sólo para dársela a una persona involucrada en la guerra de bandas) y fue arrestado y acusado de violar la Ley de Texas sobre posesión de armas. Sin embargo, un día después, agentes federales acusaron a López (un joven de 12o. grado) de la violación de la Gun-Free School Zones Act de 1990, específicamente del artículo 992 q, que consideraba un delito llevar un arma en una zona escolar, no importando que la naturaleza de la centro escolar fuera pública, privada o parroquial $^{31}$.

En la Apelación, López alegó la falta de autoridad y competencia del Congreso de los Estados Unidos de América para legislar la posesión de armas a nivel local, lo que fue así sostenido por el Tribunal de Apelaciones del Quinto Circuito, quien efectivamente consideró que el Congreso Federal carecía de esta competencia bajo la Cláusula de Comercio.

El asunto llegó finalmente al Tribunal Supremo Americano, quien el 26 de abril de 1995 decidió el caso López en una muy discutida resolución con cinco votos a favor y cuatro en contra. El Magistrado Rehnquist (Chief Justice por aquel entonces) elaboró la opinión mayoritaria de la Corte en virtud de la cual se declaró inconstitucional la Gun-free School Zones Act de 1990. La tesis fundamental en que se basó el fallo era que el Congreso carecía de competencia para regular esa cuestión, tal y como lo había alegado López y como también había sostenido el Tribunal de Apelaciones ${ }^{32}$.

Debe precisarse que la forma en la que nace el federalismo norteamericano tiene mucho que ver en la percepción que se plasma en Lopez. Si bien la genera-

n. ${ }^{\circ} 3$, Verano 1996, pp. 109-126; Kommers, Donald et al. American Constitutional Law: essays, cases and comparative notes, cap. 7, Rowman and Littlefield Publishers, 2010. De ahí surge, en parte Barnett, Randy. Restoring the lost constitution, not the constitution in exile, Washington: Georgetown Law Faculty Publications, 2006.

30 Gibbons v. Ogden, 22 US 1 (1824) es el primer caso en el que el Tribunal deja sentado la posibilidad del Congreso federal de legislar en materia de comercio intraestatal.

31514 US (549) 1995, p. 551.

32514 US (549) 1995, p. 549. 
ción de los Padres Fundadores de la Constitución de los Estados Unidos reconocía la facultad de los Estados y establecía que el gobierno federal sólo tenía aquellos poderes reconocidos expresamente en la Constitución (artículo 1), lo cierto es que lo que se pretendía en la práctica era la consolidación de un gobierno federal fuerte, que conviviera con la soberanía reconocida a los Estados. Éstos últimos, por su parte, conservaban numerosos poderes residuales, que al no entregarse a la Federación quedaban fuera de sus competencias. Finalmente, el texto constitucional contenía solo un pacto mínimo entre federalistas y antifederalistas, por lo que el TS de Marshall se encargaría de delinear las competencias de la federación para el naciente Estado norteamericano ${ }^{33}$.

Debido a esta circunstancia, en Lopez existe una fuerte discusión sobre las competencias que corresponden al Congreso Federal, y que no deben ser «sustraídas» de la esfera competencial de los Estados. Al final, la decisión versa sobre eso: que el Congreso se ha autoatribuido una competencia legislativa que sólo le está reconocida a los Estados. La consecuencia no es solo una limitación de las esferas competenciales del Congreso Federal, sino que lo que interesa es el trasfondo que justifica dicha decisión. La sentencia considera que la regulación contenida en la Gun-free School Zones Act de 1990 no es una competencia que corresponda al Congreso Federal porque la misma no está amparada bajo la commerce clause. El Supremo considera que la regulación de la posesión de un arma (que tiene naturaleza de libertad) corresponde al Estado federal, y por tanto le reconoce al legislador estatal la competencia sobre esta materia. Es, el fondo, un pronunciamiento que constituye una reivindicación de las competencias de los Estados. Una vuelta a la interpretación textual de la Constitución ${ }^{34}$.

El caso que se estudia fue el primero en 60 años en declarar inconstitucional una Ley federal dictada al amparo de la cláusula de comercio y dejó sentada la nueva visión del TS respecto a la forma en la que debe interpretarse la Constitución cuando se enfrente a casos en los que el federalismo y su marco de desarrollo estén presentes. El caso pertenece a lo que se ha llamado el «Nuevo Textualismo» ${ }^{35}$ en el que está imbuido el máximo tribunal norteamericano y que ha dado lugar a un «Nuevo Federalismo» ${ }^{36}$, que puede describirse básicamente como una «vuelta» al marco previsto por los Padres Fundadores ${ }^{37}$ para el modelo de federación, aunque, en verdad, no está del todo clara la verdadera relación de ese «Nuevo Federalismo» con la idea de federalismo de los Padres Fundadores.

33 En relación a la ideología del federalismo, el interesante libro de LaCroix, Alison. The ideological origins of American Federalism, Cambridge, Mss: Bellknap, Harvard University Press, 2010.

34 Véase Manning, J. F., «What divides Textualist from Purposivists?», en Columbia Law Review, vol. 70, n. $^{\circ} 106,2006$

35 Manning, J. F., «Federalism and the generality problem...», pp. 2013-2020.

36 La Croix, op. cit. epílogo; MANNING, J. F., «Federalism and the generality problem... », pp. 2020-2036.

37 Por lo que respecta a la visión de los Padres Fundadores, se encuentra una visión crítica en, AcKerMAN, B., The Failure of the Founding Fathers: Jeffeerson, Marshall and the rise of presidential democracy, Cambridge, Mss: Bellknap, Harvard University Press, 2005. 
Baste señalar algunos detalles de la sentencia para hacerse una idea clara de esta visión dada por el Tribunal Supremo. Así, por ejemplo, la sección 992(q) de la referida legislación trataba de evitar la violencia que se podía generar con la presencia de armas de fuego en escuelas e indicaba, además, que dicha violencia escolar podría proyectarse en distintos ámbitos e, incluso, afectar a la economía estadounidense. Se puede cuestionar lo forzado del argumento del Gobierno, pero habrá que reconocer, no obstante, la justificación basada en las consecuencias (sociales, económicas y culturales) que la seguridad en una escuela conlleva.

Sin embargo, la opinión mayoritaria encuadra el caso en una visión abstracta y genérica, de manera ciertamente sesgada. El Tribunal Supremo Norteamericano deja a un lado las peculiaridades del caso en cuanto a los objetivos que la legislación persigue, y se dispone a juzgarlo desde una perspectiva que tiene su eje en el conflicto de competencias. No importa que se trate de escuelas, ni importa que se trate de niños o jóvenes cuya seguridad se trata de proteger: lo que importa, antes y después de todo, es la competencia, lo que, por supuesto - según el TS - viene dado por el texto de la Constitución. Esta abstracción resulta de la liberación de los hechos y la vuelta a las palabras. La autoridad que emitió el acto debe ser competente y conforme a esa competencia, debe emitir su statute. Resuelve el Supremo Americano: «The possession of a gun in a local school zone is in no sense an economic activity that might, through repetition elsewhere, substantially affect any sort of interstate commerce. Respondent was a local student at a local school; there is no indication that he had recently moved in interstate commerce, and there is no requirement that his possession of the firearm have any concrete tie to interstate commerce» ${ }^{38}$.

Hasta Lopez, el TS había establecido tres razones en las que un acto jurídico puede ser efectivamente amparada por la commerce clause y, por ende, queda bajo la competencia del Congreso de los Estados Unidos, razones que son las siguientes: 1.Que se regule el uso de las vías del comercio interestatal; 2. Los medios, personas o cosas objeto de regulación sean susceptibles de comercio interestatal; 3. Las actividades (intraestatales) reguladas tengan una sustancial relación con el comercio interestatal ${ }^{39}$.

Por supuesto que los dos primeros aspectos no se daban en el caso Lopez, por lo que la sentencia se centraba en saber si el caso podía ser encuadrado constitucionalmente como un caso en el que la actividad llevada a cabo por Alfonso Lopez era una actividad que sustancialmente incidiera en las relaciones de comercio entre los distintos Estados. El Tribunal de Apelaciones advertía que no era así; la Gun-free School Zones Act no tenía el respaldo (legislativo) que sustentaba a otras leyes que apoyan esa relación de la actividad regulada con el comercio de los Estados. El argumento, en este sentido, fue ratificado por el Tribunal de Apela- 
ciones, que sostuvo que en la Ley no está explícita (ni en el procedimiento legislativo se discutió plenamente) la relación entre la regulación efectuada mediante el statute y la commerce clause en alguno de los tres campos antes mencionados. El Tribunal de Apelaciones determinó que los precedentes hacen pensar en que el Congreso debe aportar esa conexión entre el interés comercial y el statute, ya que las leyes sobre armas de fuego que adoptadas hasta entonces «habían establecido esa relación en audiencias de comisiones o en reportes». El Congreso no lo hizo así con la Gun-Free School Zones Act ${ }^{40}$, aunque también alegó que no debía hacerlo — argumento éste aceptado en última instancia por el Tribunal Supremo-.

En resumen, el Tribunal Supremo de los Estados Unidos le da la razón a Lopez en base una reinterpretación distinta del federalismo norteamericano, en el sentido de que el artículo 1 sección 8 de la Constitución Americana ya no sería una «carta abierta» a través de la cual el Congreso de los Estados Unidos podría legislar en la materia y proporción que considerase oportuno. La opinión mayoritaria del TS vuelve sus ojos al argumento del federalismo de soberanía dual, en el que los Estados son los verdaderos representantes de esa soberanía, quienes, de forma extraordinaria, ceden una parte a la federación. La infinita competencia recae en los Estados, dice el Tribunal, siguiendo a Madison ${ }^{41}$. Es en ellos, sostiene, en quienes se encuentra la facultad residual cuando la competencia no se le ha reconocido a la federación, pero las competencias expresamente reconocidas a ésta son pocas y definidas.

Lopez supone una interpretación restringida de la commerce clause. Parece un manifiesto contra los excesos de la federación, lo que no se corresponde con la realidad; en gran parte, porque el modo en que los Estados logran un equilibrio y una protección contra una eventual acción en su perjuicio por parte de la federación no se opera a través de la delimitación de competencias de la federación ${ }^{42}$.

Lopez evidencia un cambio en el TS, inspirado a partir del pensamiento de una interpretación original de la Constitución (cuyo máximo impulsor era el magistrado Scalia ${ }^{43}$ ), pero que no acaba de encontrar entre sus seguidores un camino común. Se encuentran al final del camino, pero el túnel que les conduce a dicho final es de diferente especie, puesto que unos son más textualistas que otros ${ }^{44}$.

En cuanto a los jueces que disienten en Lopez, todos coinciden en identificar a la Ley como importante debido a los fines que persigue. Dice el Magistrado Stevens: «The welfare of our future "Commerce with foreign Nations, and among

40 PAlmer y LAVERTY, «The impact of Lopez on intergovernmental relations: a preliminary assesstment», en Publius, vol. 26, n. ${ }^{\circ} 3$, Verano 1996, p. 110.

41 Hamilton et al., «The federalist papers», n. ${ }^{\circ}$ 45, New York, Palgrave MacMillan, 2009.

42 Véase Manning, J., «Federalism and the generality problem...», pp. 2061-2065.

43 En este sentido el excelente trabajo de Ch. E. SMITH: Justice Antonin Scalia and the Supreme Court's conservative moment, Westport, Conn: Praeger, 1994.

44 Un análisis puntual lo hace Rey, F., «United States v. Lopez y el "Nuevo Federalismo” Norteamericano", en Revista Española de derecho constitucional, año 17, n. ${ }^{\circ}$ 51, Septiembre-Diciembre 1997, p. 289, sobre el entendimiento del Magistrado Thomas respecto de la cláusula de comercio. 
the several States”, U. S. Const., Art. I, $\S 8, \mathrm{cl} .3$, is vitally dependent on the character of the education of our children ${ }^{45}$. Y también el Magistrado Souter: «... under commerce, as under due process, adoption of rational basis review expressed the recognition that the Court had no sustainable basis for subjecting economic regulation as such to judicial policy judgments, and for the past half century the Court has no more turned back in the direction of formalistic Commerce Clause review (as in deciding whether regulation of commerce was sufficiently direct) than it has inclined toward reasserting the substantive authority of Lochner due process (as in the inflated protection of contractual autonomy)...» ${ }^{46}$.

El mismo magistrado Souter se pregunta por la actitud del TS, en una clara referencia al caso Lochner ${ }^{47}$, expresando implícitamente un cuestionamiento sin respuesta lógica cuando afirma: «.... it seems fair to ask whether the step taken by the Court today does anything but portend a return to the untenable jurisprudence from which the Court extricated itself almost 60 years ago. The answer is not reassuring. To be sure, the occasion for today's decision reflects the century's end, not its beginning. But if it seems anomalous that the Congress of the United States has taken to regulating school yards, the Act in question is still probably no more remarkable than state regulation of bake shops 90 years ago» ${ }^{48}$.

La importancia del cambio en la interpretación constitucional dada en Lopez queda subrayada por el magistrado Breyer cuando comienza su disenso con la siguiente reflexión «In my view, the statute falls well within the scope of the commerce power as this Court has understood that power over the last half century» ${ }^{49}$.

Sin duda, el magistrado Breyer es quien realiza un argumento mucho más razonado. Basa su desacuerdo en la posibilidad del Congreso de encontrar una rational basis con la cual sustentar la ley discutida en Lopez ${ }^{50}$.

Breyer sigue una línea en su argumento: ¿qué significa que los alumnos tengan armas en la escuela? Al efecto, aporta cifras y un apéndice a la sentencia sobre el número de alumnos que ocasionalmente portan un arma en las escuelas de los Estados Unidos de América. Bajo esta premisa, Breyer concluye que la posesión de armas en una escuela es una cuestión importante que puede incidir de forma significativa en la calidad de la educación. Después, Breyer da un segundo salto al relacionar la calidad de la educación con la economía: «Having found that guns in schools significantly undermine the quality of education in our Nation's

45514 US (549) 1995, p. 602.

46514 US (549) 1995, p. 607

47 Lochner v. New York, 198 U.S. 45 (1905).

48514 US (549) 1995, p. 608

49514 US (549) 1995, p. 615

50 Véase Fallon JR., R., «Strict Judicial Scrutinity» en 54 UCLA Law Review, 2007, 1267-1337; y JACKSON, V., "Constitutional Law in an Age of Proportionality», en Yale Law Journal, vol. 124, n. ${ }^{\circ}$ 8, Junio 2005, pp. 2680-3203. 
classrooms, Congress could also have found, given the effect of education upon interstate and foreign commerce, that gun-related violence in and around schools is a commercial, as well as a human, problem $»^{51}$.

El magistrado establece que la violencia generada por la posesión de armas en las escuelas es un problema de naturaleza económica y por ende comercial. Proporciona un dato que parece fundamentar muy bien su argumento: casi un cuarto del crecimiento económico de los Estados Unidos se debe, directamente, al nivel de la escolaridad ${ }^{52}$; y así, va dando cifras Breyer, relativas a los tipos de trabajo que requiere el mercado, los cuales han cambiado, y que necesariamente se tiene que ajustar la calidad educativa, la planificación corporativa y su relación con la educación, basando sus decisiones de establecimiento en la existencia o no de una determinada fuerza de trabajo ${ }^{53}$.

Con ello, Breyer parece que cierra la discusión en favor de la inminente relación de la educación-economía-commerce clause. Todo el argumento de Breyer gira alrededor de ello: es un asunto que cae dentro de la comercial clause y, por ende, es el Congreso Federal el organismo competente para la legislar sobre el mismo y no los Estados.

La conclusión de Breyer es afirmativa en ambas cuestiones, porque la relación entre la calidad educativa y la economía se basa en la serie de cifras que aporta; y esa calidad educativa depende, en gran parte, de que no existan armas en la escuela. Pero, ¿por qué el afán de Breyer de dar cifras? La respuesta se encuentra en el argumento que hemos analizado anteriormente, en el sentido de que el Congreso no había aportado los fundamentos que hacen presumible la relación intrínsecamente económica entre portar armas y el comercio entre los Estados. Breyer asume la responsabilidad del legislador y trata de apelar a los propósitos de la legislación como la verdadera fuente a considerar. Piensa, como intencionalista ${ }^{54}$, que la manera de enfrentar el problema no sólo es saber si el caso concreto merece o no ser un caso de competencia, sino saber si el propósito del legislador es bueno o no. Breyer sigue la estela de los casos del New Deal ${ }^{55}$ en los que se da una clara deferencia al legislador y se analiza si existe o no una rational basis para que el legislador emita esa ley ${ }^{56}$.

Lo que el magistrado pasa por alto es que el bloque conservador ya había desechado esa posibilidad, es decir, en sí, Breyer disiente en cuanto al fondo del asunto (la inconstitucionalidad del statute), pero no ataca los argumentos de la

51514 US (549) 1995, p. 620.

52514 US (549) 1995, p. 620.

53514 US (549) 1995, p. 622.

54 Brillante la forma en que Manning ha expuesto las diferencias entre intencionalismo y textualismo. MANNING, John, «Federalism and the generality problem...», pp. 2009-2020.

55 Principalmente N.R.L.B. v. Jones \& Laughlin Steel Corp. 301 US 1 (1937), United States v. Darby, 312 U. S. 100 (1941) y Wickard v. Filburn, 317 U. S. 111 (1942).

56 En la misma línea puede verse Guastini, R., Estudios de Teoría Constitucional, México: Fontamara-UNAM, 2001, pp. 255-274. 
mayoría. Ellos son textualistas y Breyer un intencionalista, que lo que quiere es apelar a la esencial rational basis de la Ley que, obviamente, se encuentra o se puede encontrar. En su razonmiento, Breyer analiza el problema desde una perspectiva distinta: se abstrae para interpretar conforme a la Constitución; el bloque conservador optó por tomar lo que le convenía (el texto de la Constitución) y establecer su criterio (se abstraen de los hechos para basarse en el texto). Es, en el fondo, una guerra ideológica: de un lado, quienes reconocen la necesidad de regulación por parte del Estado y, por el otro, los que quieren dejar en su espacio mínimo esa capacidad reguladora del Estado federal. Es una cuestión de más o menos Estado. Una vez más, aquella clásica contradicción entre libertad e igualdad. Unos los que buscan que haya más libertad y otros los que consideran que con la acción del Gobierno, se puede alcanzar más igualdad. Al final, un binomio contradictorio $^{57}$.

\section{La cláusula de comercio}

Como ya mencionamos, la cláusula de comercio ha sido importantísima en la vida constitucional de los Estados Unidos de América. Lo que el TS ha hecho, desde el Tribunal Marshall y hasta Lopez es ir expandiendo y contrayendo esa cláusula.

En Gibbons ${ }^{58}$, el Tribunal Marshall reconoció la capacidad del Congreso para legislar con base en la commerce clause, la cual experimentó su punto más extremo en Wickard, donde se declaró aplicable un statute que prohibía cierta producción de trigo, a pesar de que la persona a la que se le aplicaba lo cultivaba para su propio consumo. El Supremo reconoció una relación entre la producción para el consumo de trigo y sus efectos en el mercado. Señaló el TS en Wickard: «The effect of the statute before us is to restrict the amount which may be produced for market and the extent as well to which one may forestall resort to the market by producing to meet his own needs. That appellee's own contribution to the demand for wheat may be trivial by itself is not enough to remove him from the scope of federal regulation where, as here, his contribution, taken together with that of many others similarly situated, is far from trivial... ${ }^{59}$.

Así, el Tribunal llegaba al punto más amplio en relación con la interpretación de la cláusula de comercio. Sin embargo, esta aproximación no fue siempre así, porque es solo a partir de Darby cuando el TS comienza a reconocer la capacidad del Congreso para legislar «en todo su alcance posible» ${ }^{60}$ en base a la cláusula de comercio. Es en Darby donde se produce el punto de inflexión a partir del

\footnotetext{
57 Bobbio, N., Teoría General de la Política, Madrid: Trotta, p. 455.

58 Gibbons v. Ogden, 9 Wheat. 1, 189-190 (1824).

59 Wickard v. Filburn, 317 U. S. 111 (1942), p. 128

60 United States v. Darby, 312 U. S. 100 (1941).
} 
cual el TS echa abajo toda las limitaciones previas (con el caso Lochner) y configura una nueva concepción de la commerce clause en tres aspectos: en primer lugar, la idea de un federalismo dual se ve abandonada y la décima enmienda se reduce a una perogrullada; en segundo lugar, el Congreso puede regular prácticamente todo, una vez que entre dentro del comercio; y tercero, el Congreso puede regular actividades intraestatales demostrando efectos sustanciales en el comercio intraestatal, pudiendo ser esa demostración respecto de distintos signos — por ejemplo, un agregado de pequeñas transacciones (Wickard) ${ }^{61}$.

En Lopez, el Tribunal cambia el entendimiento de la commerce clause. Pare ello, Rehnquist, después de hacer un recuento de la commerce clause, dice: «First, we have upheld a wide variety of congressional Acts regulating intrastate economic activity where we have concluded that the activity substantially affected interstate commerce... Where economic activity substantially affects interstate commerce, legislation regulating that activity will be sustaine... Section 922(q) is a criminal statute that by its terms has nothing to do with «commerce» or any sort of economic enterprise, however broadly one might define those terms ${ }^{62}$.

La mayoría del Supremo norteamericano define así el cambio de canon: Although Justice Breyer argues that acceptance of the Government's rationales would not authorize a general federal police power, he is unable to identify any activity that the States may regulate but Congress may not» ${ }^{63}$.

El Chief Justice y la mayoría que se adhirió a su opinión son de la idea de que la commerce clause debe ser más restrictiva, pues dejarla abierta permite que exista una falta de distinción entre los campos a regular por los Estados y los que tiene la federación. El Tribunal vuelve a entender que una interpretación abierta de la commerce clause tiene como resultado un debilitamiento de las entidades federativas, en tanto en cuanto dicha cláusula se convierte en un competencia transversal que habilita al Legislador federal en casi cualquier ámbito.

Rehnquist entona un extraño mea culpa para justificar el cambio de posición del Tribunal. Sostiene: «Admittedly, some of our prior cases have taken long steps down that road, giving great deference to congressional actio... The broad language in these opinions has suggested the possibility of additional expansion, but we decline here to proceed any further. To do so would require us to conclude that the Constitution's enumeration of powers does not presuppose something not enumerated, cf. Gibbons v. Ogden, supra, at 195, and that there never will be a distinction between what is truly national and what is truly local, ... This we are unwilling to do» ${ }^{64}$.

La transcripción anterior no deja lugar a dudas: el TS está dejando atrás su antiguo entendimiento de las facultades del Congreso - a través de la commerce

61 Farber et al., op. cit., p. 859.

62 Ídem.

63514 US (549), 1995, p. 564.

64514 US (549), 1995, pp. 567-568. 
clause - y lo está haciendo contraviniendo precedentes importantísimos, como McCulloch v. Maryland ${ }^{65}$, en el que el Tribunal estableció que existen poderes implícitos, no enumerados, que el Congreso tiene y que provienen de la misma Constitución (lo cual niega Rehnquist en la parte transcrita). Lo que la mayoría conservadora del Tribunal Supremo lleva a cabo es apegarse al texto constitucional de manera estrechísima: no hay disposición expresa, no hay competencia. Esa era exactamente la posición del Estado de Maryland en el caso supracitado: la visión originalista de la Constitución debía prevalecer. El Tribunal sostiene que esa visión originalista (mejor dicho, textualista) debe retomarse. Los anteriores criterios, sostiene, «han dado pasos importantes» en una dirección equivocada. Que el gobierno federal tiene poderes mínimos (y con ello una posibilidad de regulación también mínima) es la lectura «correcta».

\section{Nuevo textualismo / Nuevo Federalismo}

Desde el New Deal, el Supremo había dejado en claro la competencia casi sin límites del Congreso en materia de comercio. La commerce clause ha sido - desdel Tribunal Marshall_ - la piedra sobre la que ha forjado el Congreso de los Estados Unidos de América su funcionamiento y sobre la que se ha basado la capacidad gubernativa de establecer impuestos y realizar gastos del gobierno federal en relación con los Estados.

Por ello, Lopez no es un caso menor; en base a él se está reconfigurando la cláusula a partir de la cual operan en gran parte las facultades del gobierno federal. Esto no es sino una maniobra más en la conocida guerra de los magistrados conservadores en contra de Roe v. Wade (la decisión del TS que reconoció la legalidad del aborto). No es que estén interesados en las competencias de los Estados, lo que sucede es que esa interpretación (menos gobierno federal, más Estados) es a la que conduce su visión originalista de la Constitución ${ }^{66}$, y ella les permitirá revertir criterios con los que no se encuentran a gusto, así como tener un control de armas menos estricto y más favorable hacia grupos como la Asociación Nacional del Rifle, más allá de los fines de la legislación, como se demuestra en el propio caso Lopez.

Recientemente se ha defendido el «Nuevo Originalismo», con el magistrado Scalia como punta de lanza, como una nueva forma de argumentar, aunque en

65 Es el famoso caso de la creación de un Banco Central, en el que el Supremo echó por tierra la imposición de gravámenes a dicho Banco y sostuvo que si bien no está prevista explícitamente esa facultad, ésta se podía inferir de la Constitución. Cfr. McCulloch v. Maryland 17 US (316) 1819.

66 Aunque, como apunta Manning, es muy peculiar que en la sentencia de Lopez el TS no base su decisión en ningún fundamento, sino lo realice en documentos preconstitucionales, en las discusiones de la Asamblea constituyente y en documentos de la antigua Confederación de Estados. MANNING, J., «Federalism and the generality problem...», p. 2006. 
esencia termina siendo un textualismo refinado ${ }^{67}$, con diccionarios y fuentes etimológicas como su base, pero que muy poco se distingue de ese textualismo ${ }^{68}$, que reclama una interpretación constitucional empezando por la letra escrita: «El texto es la ley, y es el texto el que debe ser observado» ${ }^{69}$.

Es por eso que se produce un «overruling» a favor de los Estados. El Tribunal Lopez interpreta la Constitución para acotar el poder del Congreso Federal. Antes del reconocimiento de la constitucionalidad de las leyes del New Deal, el TS había previsto un federalismo basado en las división radical de soberanía entre el ámbito federal y el ámbito estatal (federalismo dual), proyectado en la delimitación del Congreso federal a los poderes exactamente enumerados en la Constitución. A partir del New Deal, se deja de lado esta percepción, para dar paso a las reformas de Roosevelt que impulsaron la economía. El TS acepta su papel no intervencionista, pero con la conocida vigilancia que dejó entrever en Carolen Products $^{70}$. A partir de los casos Printz ${ }^{71}$ y New York ${ }^{72}$ el TS retoma un concepto de federalismo que, así como el textualismo, se ha venido denominando «Nuevo Federalismo». Este consiste en una interpretación textualista/originalista de la Constitución, criticando tres puntos importantes de la visión hasta ahora llevada a cabo por el TS en esta materia: 1. El federalismo como reflejo de compromisos; 2. El federalismo como la adopción de una serie de medidas particulares que reflejan el objetivo de fondo (como una norma establecida libremente); 3. Imponer el valor del federalismo en abstracto (abstraerse del caso concreto que se analiza).

La visión de este Nuevo Federalismo es que los Estados son quienes tienen las mayores y originales competencias y la Federación tiene las que expresamente le reconoce la Constitución — con el aderezo de que la Constitución es la que literalmente las señala.

Pero ¿qué pasa y qué pasará con los casos en los que exista un conflicto entre ese Nuevo Federalismo (conservador y que trata de quitar al Estado federal su actividad reguladora en los Estados Unidos de América) y otros principios constitucionales o, incluso, con los derechos humanos? ¿Cuál es el peso de ese sistema federal de protección de la soberanía de los Estados, como lo entiende el Supremo Americano, en una ponderación de principios constitucionales? ${ }^{73} \mathrm{~A}$ partir de Lopez, el canon parece inclinarse a favor de la protección de ese nuevo federalismo: lo importante es la interpretación originalista de la Constitución, lo que debe llevar a «respetar» la facultad originaria del Estado, porque la Federación solo

67 Kommers, D. et al., American Constitutional Law: essays, cases and comparative notes, p. 39.

68 Posner, R. A., How judges think, Cambridge, Mass: Harvard University Press, 2008.

69 Scalia, A., A Matter of interpretation: Federal Courts and the Law, Princeton: Princeton University Press, 1997, p. 22.

70 En la famosa nota cuatro, podría interpretarse como una declaración del TS a mantenerse no intervencionista, pero cuidadosa en los casos de discriminación.

71 Printz v. United States 521 US 898 (1997).

72 New York v. United States 505 US 144 (1992).

73 Alexy, R., Teoría de los derechos fundamentales, CEC, Madrid, 1993. 
tiene aquellas competencias que la Constitución prevea, y que, textualmente, son muy escasas a favor de la Federación ${ }^{74}$.

Y es aquí donde encuentran relación Lopez y Heller. El Magistrado Rehnquist lo dijo en su momento, sobre cómo ha cambiado esa concepción de la commerce clause, para convertir la autoridad del Congreso (federal): «under the Commerce Clause to a general police power of the sort retained by the states». Es decir, lo que el TS está poniendo de manifiesto es que la facultad legislativa dada al Congreso se ha convertido, según sus palabras, en una política, y que ésta, en todo caso, debe encontrar su límite en los Estados. En ambos casos, el límite soberanía estatal — se impone a los fines y principios en discusión.

El TS no quiere dejar de lado esa noción de abstraerse del caso y ver los fines de la Ley para, a partir de ellos, saber si existe una rational basis con la que se pueda compatibilizar la acción legislativa. El Tribunal insistirá en la noción textual de la Constitución, que ofrecía un gobierno federal que protegería a los Estados de naciones extranjeras y que tendría la capacidad de dirimir las disputas que surgieran entre ellos.

Por eso es que en Lopez la interpretación de la Segunda Enmienda es reduccionista: la Constitución no dice claramente que el Estado puede restringir esa libertad del individuo a portar armas.

¿Pudo el TS interpretar Lopez de una manera distinta? La respuesta es sí $^{75}$; aunque queda claro que en Lopez, la seguridad pública y el derecho a la educación quedaron de lado. Es un autoconvencimiento constitucional en sede del Tribunal Supremo. Lo importante no son los fines, sino las palabras. Si el legislador y el constituyente sostuvieron tal o cual cosa, eso no es sino muestra de un compromiso y ese compromiso debe respetarse. El compromiso no se encuentra en los fines de la ley; se encuentra en las palabras expresadas. Por ello, educación y seguridad pública son dejados de lado para dar paso a un reforzamiento de los poderes de los Estados.

¿Significa que todo principio constitucional o que los derechos fundamentales que vayan en contra de ese Nuevo Federalismo tienen perdida la batalla? Tendrán perdida la batalla en tres sentidos: a. En el sentido de que el autoconvencimiento constitucional en que ha entrado el TS Americano no deja lugar a la discusión cuando hay que salvaguardar alguna competencia de los Estados; b. Si apelan a los fines de ese principio o a los fines de la ley o resolución en que se

74 Ya en Garcia v. San Antonio Metropolitan Transit Authority (1985).10 el TS había previsto cómo proteger a los Estados de los excesos de la Federación. En este caso. el TS había previsto que es en el proceso político nacional en donde los Estados deben buscar la protección sus intereses en el sistema federal. Garcia se fortaleció en Baker v. South Carolina (1988), 11 declarando el TS que no había prohibición para proteger a la imposición de impuestos a bonos municipales bajo la X enmienda. El TS reconocía el poder amplio de la federación.

75 Pudo haber mirado a la X enmienda para encontrar la salvaguarda de los Estados. Véase Palmer y Laverty, op. cit., p. 125. 
basen; c. Si esos principios se encuentran reflejados en competencias federales, es decir, si son a través de actos de la federación o de obligaciones contraídas por la federación, como se han expresado.

Ese autoconvencimiento constitucional no se superará sino con remedios directos: la discusión de la importancia de los principios en disputa. La respuesta más efectiva (desde el TS) a este ataque originalista de la interpretación de la constitución, pareciera ser la clásica: mirar a los fines de la Constitución y los fines de las Enmiendas ${ }^{76}$.

\section{HELLER: VOLVIENDO A LA SEGUNDA ENMIENDA}

En una decisión de cinco votos contra cuatro, el Tribunal Supremo de los Estados Unidos de América decidió el caso District of Columbia v. Heller (Heller, de aquí en adelante), siendo el ponente de la resolución el magistrado conservador Antonin Scalia.

Heller es el punto de arranque de moda de toda la discusión sobre la regulación para tener y portar armas de fuego en los Estados Unidos de América. La discusión ha florecido en los últimos tiempos, especialmente por las matanzas en Orlando y Dallas en las que el derecho reconocido en la Segunda Enmienda a portar armas de fuego ha facilitado el uso de armas de asalto que han acabado con la vida de cientos de personas. Como antes se afirmó, esos casos no son excepcionales, sino que el uso de armas de fuego contra ciudadanos es cada día más usual ${ }^{77}$.

La lectura de Heller, como todos los grandes asuntos resueltos por el Tribunal Supremo Americano, debe matizarse con el voto particular de la disidencia. Heller significa el triunfo de una visión textualista y originalista de la constitución americana, en lo que a la regulación de tenencia y porte de armas se refiere, pero tan importante es la opinión mayoritaria de Heller como la visión minoritaria.

La cuestión central en Heller era determinar si la prohibición para poseer armas violaba o no la Segunda Enmienda ${ }^{78}$. Dick Heller, un policía que estaba autorizado para portar armas durante el cumplimiento de su deber, solicitó un certificado de registro para tener un arma de fuego en casa, pero el Distrito de

76 A este respecto pienso que, de llegarse a dar el caso, será trágica la caída de la reforma sanitaria del Presidente Obama. Es una lucha desigual, porque es en distintos caminos, en distintas arenas. Es una lucha entre letras y espíritu. Cfr. MANNING, J., «Federalism and the generality problem...», pp. 2009-2025.

77 Véase «Dallas tragedy proves a good guy with a gun shouldn't be the only thing that stops a bad guy with a gun» en Los Angeles Times, Julio 11 de 2016, revisado el 13 de julio de 2016, disponible en http:// www.latimes.com/politics/la-pol-ca-skelton-dallas-shooting-gun-control-20160711-snap-story.html; también «Orlando, Dallas similarities include military-style rifles», en Orlando Sentinel, Julio de 2016, revisado el 13/07/2016, disponible en http://www.orlandosentinel.com/news/breaking-news/os-dallas-shooting-gunorlando-20160708-story.html

78 District of Columbia v. Heller. 554. U.S. (2008) p. 1. 
Columbia se lo negó ${ }^{79}$ de conformidad con las leyes que criminalizaban la posesión de armas no registradas.

Si se lee con cuidado la opinión mayoritaria, en una primera parte Scalia detalla los pormenores textuales de la Segunda Enmienda ${ }^{80}$. En ella hace hincapié en el contexto del que surge la tenencia de armas y otorga una inusual atención al significado de los dos verbos (portar y tener) ${ }^{81}$, que, según su visión, tienen una connotación distinta.

Sin embargo, donde el alegato de Scalia es menos convincente es en los fines para los cuales se protege la tenencia de las armas de fuego ${ }^{82}$, siendo en ocasiones su argumento de naturaleza circular ${ }^{83}$. Al estar analizando los fines de la protección de ese derecho a portar armas vuelve a hacer hincapié en que es un derecho y que tiene ciertos límites, lo que ya había advertido en un apartado anterior.

La Segunda Enmienda enfatiza los fines de esa tenencia, que son para la protección de la nación y de la «seguridad de un Estado libre» ${ }^{84}$. Este es el punto de mayor discusión entre las posiciones dentro del TS Americano, que se reproduce en la sociedad estadounidense.

El derecho a tener armas no solo tiene como presupuesto un fin de seguridad, sino que éste, a su vez, es un límite que tendría que ser infranqueable. La opinión mayoritaria del TS liderado por Scalia es contraria, pues en Heller, cuando empieza a analizar esos límites, se centró en la discusión de si se trata de un derecho preexistente o es un derecho que nace a partir de la aprobación de la Segunda Enmienda ${ }^{85}$.

Y la interpretación no termina de ser peculiar porque, por ejemplo, cuando aspira a definir a qué se refiere la Enmienda con un ejército debidamente regulado, termina por afirmar que: «the adjective "well-regulated" implies nothing more than the imposition of proper discipline and training», a efecto de afirmar que no solo se refería al ejército de los Estados Unidos, sino a un derecho de cualquier persona a formar parte de un cuerpo armado, tratando de reafirmar su posi-

79 District of Columbia v. Heller. 554. U.S. (2008) p. 2.

80 Especialmente de la p. 7 a la 18 de su opinión. Hace un alegato hipertextualista de la locución «Keep and bear arms». De hecho, comienza el texto especificando la técnica a partir de la cual interpretará la constitución en este caso concreto: «[t]he Constitution was written to be understood by the voters; its words and phrases were used in their normal and ordinary as distinguished from technical meaning». SCOTUS, op. cit., p. 3.

81 Op. cit., pp. 7-18.

82 Op. cit., pp. 19-22.

83 Sostiene: There seems to us no doubt, on the basis of both text and history, that the Second Amendment conferred an individual right to keep and bear arms. Of course the right was not unlimited, just as the First Amendment's right of free speech was not, see, e.g., United States v. Williams, 553 U. S. __ (2008). Thus, we do not read the Second Amendment to protect the right of citizens to carry arms for any sort of confrontation, just as we do not read the First Amendment to protect the right of citizens to speak for any purpose.

84 «A well regulated Militia, being necessary to the security of a free State, the right of the people to keep and bear Arms, shall not be infringed».

85 Pp. 19-22. 
ción con el argumento de que los tiranos han eliminado ejércitos «simply by taking away the people's arms, enabling a select militia or standing army to suppress political opponents» ${ }^{86}$. En otras palabras, para Scalia y para la mayoría del Supremo Americano, vedar ese derecho a tener armas es un caldo de cultivo para los tiranos. Sostiene: «It is therefore entirely sensible that the Second Amendment's prefatory clause announces the purpose for which the right was codified: to prevent elimination of the militia» ${ }^{87}$.

La interpretación roza lo risible cuando hace alusión a otros experimentos constitucionales previos a la Constitución Federal, como la Constitución de Massachusetts, que advierte un límite como el de la defensa común y que, cita el Supremo Americano, fue interpretado en su momento comparándolo con la libertad de prensa, que era ilimitada y que tenía como responsable a quien lo ejercía ${ }^{88}$.

El argumento es irrisorio porque poner bajo la misma palestra el ejercicio del derecho de la prensa y del derecho a portar armas es ya arriesgado, pues los efectos del ejercicio de ambos derechos son completamente distintos. Pero, además, porque la experiencia actual de los Estados Unidos no demuestra sino un constante ejemplo de que el uso de armas de fuego está al alcance de jóvenes que lo utilizan de manera desastrosa. Desde Virginia Tech, pasando por el Cine en Los Ángeles durante el estreno de «El Caballero Oscuro», hasta la más reciente tragedia en Orlando, se puede identificar el uso de armas de fuego contra la población civil que no tiene posibilidad de defenderse porque los resultados del ejercicio del derecho a portar armas terminan siendo letales, algo muy distinto y menos trágico que el ejercicio de la libertad de prensa.

En otras palabras, el acceso a armas de fuego de todo tipo se muestra como un problema, más que como una solución. La afirmación de la mayoría del supremo en el sentido de que existe una relación entre el surgimiento de tiranos y el no derecho a portar armas es discutible, por decir lo menos.

Los grupos que en el siglo XVIII sostenían la necesidad de asegurar que cada persona pudiera tener derecho a tener armas, portarlas y, obviamente, utilizarlas, tenían un contexto del cual se escabulle hábilmente Scalia en su proyecto avalado por la mayoría de magistrados del Tribunal Supremo en Heller: la guerra justificaba el temor de muchos a enfrentarse a personas de otro bando sin los instrumentos necesarios. Los políticos se enfrentaban a la posibilidad de no obtener los consensos necesarios para los propósitos que perseguían, pero ello no es el caso ni es el contexto actual. Scalia juega el juego del originalismo porque el contexto es el que cambia, aunque no cambia lo esencial que se desprende de su argumento:

86 P. 25.

87 Op. cit., p. 26.

88 «The liberty of the press was to be unrestrained, but he who used it was to be responsible in cases of its abuse; like the right to keep fire arms, which does not protect him who uses them for annoyance or destruction». 
que hay un derecho a portar armas. Deja de lado los fines; en última instancia, lo que importa es el texto.

Sin embargo, los magistrados Stevens y Breyer redactaron opiniones disidentes - adhiriéndose mutuamente uno a la del otro-, y a las que se sumaron Souter y Ginsburg. La de Stevens parece dar en el clavo en el inicio de su disenso, cuando sostiene que: «a conclusion that the Second Amendment protects an individual right does not tell us anything about the scope of that right» ${ }^{89}$.

Stevens es claro al afirmar que la forma en la que se aborda el análisis de la Segunda Enmienda por parte de Scalia es engañosa, porque el preámbulo es claro en cuanto a la intención de que ese derecho sea para la preservación de un Estado libre, y que en ningún momento se hace referencia a la defensa propia como un fin de la Enmienda ${ }^{90}$. La forma en que se propone la discusión condiciona la discusión misma. Cuando Scalia propone abordar primero el texto y los derechos que de él se derivan, hace sin duda más difícil que después se discutan los hechos.

Sostiene el magistrado que la forma en la que la ponencia desarrolla su argumento denigra la importancia del preámbulo de la cláusula objeto de análisis, estableciendo un debate entre derecho y no derecho, y no un debate entre el uso y posesión militar y no militar de armas, como el mismo TS había hecho en $M i$ $\| l e r^{11}$. En el fondo, la hermenéutica constitucional ortodoxa puede hacer mucho daño, se puede decir más alto pero no más claro, a saber: «The Court today tries to denigrate the importance of this clause of the Amendment by beginning its analysis with the Amendment's operative provision and returning to the preamble merely "to ensure that our reading of the operative clause is consistent with the announced purpose" ${ }^{92}$.

\section{CONCLUSIONES}

Como hemos tenido ocasión de constatar al analizar la génesis y evolución constitucional y legislativa de la Segunda Enmienda de la Constitución americana, la misma tuvo una inspiración británica pero, desde el comienzo, construyó un marco propio del complejo federalismo ascendente. Consagró un derecho sacrosanto en lo individual y fuertemente estatalizado. La evolución histórica reforzó el carácter individual del derecho, llegando mucho más allá de su conexión histórica con la milicia y la revolución americana. Sin embargo, dicha evolución,

89 Op. cit. Stevens Dissenting, p. 1.

90 Esto mismo se ve resaltado en el disenso del juez Breyer, que refiere que esa defensa propia por sí sola no fue el propósito de la enmienda, además de que, dice Breyer, la mayoría no puede sostener que es un derecho absoluto y que no puede ser regulado, por lo que tendría que demostrar la regulación es ilógica e inapropiada. Op. cit. Breyer Dissenting, p. 1.

91 Op. cit. Stevens Dissenting, p. 43.

92 Op. cit. Stevens Dissenting, p. 8. 
por el lado de la distribución vertical de competencias, vio surgir una progresiva y firme federalización de la materia en sede legislativa con una base jurídica muy débil: la clausula comercial.

Las sentencias analizadas en el presente trabajo con más profundidad (Heller y López) dictadas por el Tribunal Supremo de los Estados Unidos de América recientemente, nos ayudan a entender, de un lado, el impacto constitucional de la cuestión en sentido estricto y, de otro, el impacto del nuevo federalismo, tal y como lo está entendiendo el TS, o por mejor decir, la mayoría conservadora que lo ha dominado desde Reagan. La conclusión es clara: el TS, guiado de un «neoriginalismo» y de la un reduccionismo de la hermenéutica constitucional a la literalidad y a la ortodoxia, deja la delicada cuestión del armamento, de todos los armamentos y de todas su manifestaciones, en manos exclusiva de los Estados de la Unión. Dichas decisiones, aparte de suponer un retraso de décadas en el indiscutible avance en el control de la venta y la posesión de armas de fuego, implican la imposibilidad, a corto y medio plazo, de avances en la materia. También implican la necesaria entrada en juego de los mecanismos sociales, políticos y jurídicos de la modificación constitucional para poder volver a girar el rumbo constitucional, ciertamente de querer hacerlo de forma sostenible ante futuras mayorías conservadoras del TS. Todo ello, a su vez, nos obliga a concluir también sobre el estado de esta cuestión. Lo que hacemos de forma inmediata.

El contexto sociopolítico actual en los Estados Unidos hace impensable la aprobación de una normativa que restrinja sustantivamente la posesión de armas de fuego o una reforma constitucional pese a que, por las razones explicadas, podemos entrar en una época marcadamente liberal (a la americana) a efectos constitucionales. Ni siquiera con un nuevo gobierno Clinton, de Hillary esta vez, se daría una administración completamente liberada de los compromisos con la industria armamentística en los Estados Unidos. Sencillamente, porque, con independencia de la procedencia de los apoyos a los Clinton, el sistema de contrapoderes diseñado por los Padres Fundadores y la evolución de las (necesariamente largas) carreras de los políticos con aspiraciones a asumir posiciones de liderazgo en cualquier poder, lo hará imposible sin un cambio generacional.

Incluso, aunque el criterio sentado en Heller se pudiera revertir con una conformación más liberal del Tribunal Supremo, el problema seguiría estando circunscrito a una pobrísima base constitucional federal, la vinculada al comercio y, con ella, vinculada a puntos de venta, y millones de armas que ya se encuentran en los hogares americanos, además de políticas impulsadas por el gobierno para llenar de armas a los hogares americanos y al crimen organizado en sus distintas formas en los Estados Unidos y en otros países.

Los defensores de esta dinámica, y la inercia de la renovación de cuadros políticos, no permitirán en muchos años una regulación que prohíba o limite de manera amplia la posesión de armas de fuego. Como mucho aceptarán con reticencia una restricción para posesión de armas de asalto como la aprobada en 1994. Y no podemos olvidar que los defensores de esta dinámica son la mayoría 
de los Senadores y de los Representantes, lo fueron todos los candidatos republicanos a la presidencia de los Estados Unidos y, con distinta intensidad, los dos candidatos finalistas en las primarias del partido demócrata en 2016. Si de muestra vale un botón, véase la complejidad del tema en el hecho de que el candidato más progresista de la historia de los Estados Unidos con alguna posibilidad de ser candidato por un partido de gobierno a la Casa Blanca, Bernie Sanders, se posicionó en contra de cambiar el status quo en la materia caso de ser el Presidente. Eso, y solamente eso, provocó que Hillary Clinton nadara en esas aguas con mayor asiduidad y distinto tono del que tenía previsto inicialmente.

El tema que nos ha ocupado en estas páginas, como apuntamos en la justificación del estudio, tiene una trascendencia constitucional innegable. En él se conjugan: la seguridad pública —eje de las políticas públicas en la sociedad mundial desde el 2001-; la violencia que originan millones de armas de fuego que proliferan sin control en las calles de los Estados Unidos; derechos y libertades básicas de lo sujetos activos y pasivos de los tiroteos descontrolados o de francotiradores; la distribución vertical de competencias, dejando manifiestamente claro que la federalización de ciertas competencias es el único camino para acometer con alguna posibilidad de éxito algunas cuestiones de dimensión global. Haciendo referencia a esta última dimensión, la denuncia a la miopía del «fronterismo», la que ignora lo que pasa más allá de la frontera de los Estados Unidos al abordar este debate, no puede sino reiterarse y dimensionarse al evaluar la alucinación de pensar que la cuestión se puede resolver con un dominó de cambios a nivel estatal, como con el matrimonio homosexual o la legalización terapéutica de la marihuana.

La eventual confirmación de la llegada de Garland al Tribunal Supremo, y el presumible fortalecimiento del ala liberal del tribunal, permitirá que una sensibilidad distinta delimite el alcance de la Segunda Enmienda de los Estados Unidos de forma más acorde con los tiempos y menos con la interpretación literal originalista. Será cuestión de esperar a que asuntos como Peruta o similares lleguen a esa sede. Pero ello será simplemente abrir una nueva fase en el vaivén político-constitucional de los Estados Unidos en esta materia. La superación de este subibaja, el verdadero cambio, solamente se dará cuando los americanos superen su autosuficiencia constitucional y, evolucionen hacia una concepción más democrática la relación y los momentos históricos del poder constituyente y el poder constituido.

\section{$* * *$}

\section{TITLE: Gun control in the New US Federalism}

AвSTRACT: This paper analyses the main constitutional dimensions of gun possession in the United States of America: individual rights and freedoms, public safety and the distribution of powers between the Federation and the States. Our study focuses mainly in constitutional bistory and constitutional case law interpretation, especially in Heller and Lopez cases. We conclude that this case law breaks dramatically with 
the progressive federalization of competencies developed because of the commercial clause. Furthermore, we see no means for a constitutional development in favor of gun control.

RESUMEN: En el presente trabajo se analizan las principales dimensiones constitucionales de la posesión de armas de fuego en los Estados Unidos de América: los derechos y libertades individuales, la seguridad pública, y la distribución de competencias entre la Federación y los Estados. En este análisis acudimos principalmente a la bistoria constitucional y a la bermenéutica de la doctrina constitucional del Tribunal Supremo americano, especialmente de las sentencias Heller y López. Se puede concluir que dicha jurisprudencia rompe dramáticamente con la progresiva federalización competencial desarrollada sobre la base de la cláusula comercial. A mayor abundamente, consideramos que actualmente no existen razones para un desarrollo constitucional favorable al control de armas.

KEY WORDS: Gun possession, Second Amendment, constitutional originalism.

Palabras Clave: Posesión de armas, Segunda Enmienda, originalismo constitucional.

FECHA DE RECEPCIÓN: 05.06.2016

FECHA DE ACEPTACIÓN: 27.07.2016 